\title{
Pneumatosis Intestinalis and Pneumomediastinum in Patients With Granulomatosis With Polyangiitis
}

\author{
Aran Kim, M.D. ${ }^{1}$, Jung Hee Koh, M.D., Ph.D. ${ }^{2}$ \\ ${ }^{1}$ Division of Rheumatology, Department of Internal Medicine, Pusan National University Hospital, Busan, ${ }^{2}$ Division of Rheumatology, \\ Department of Internal Medicine, Bucheon St. Mary's Hospital, College of Medicine, The Catholic University of Korea, Seoul, Korea
}

A 66-year-old male patient presented with dyspnea and blood tinged sputum for 2 weeks. He had a 4-month history of granulomatosis with polyangiitis, with proteinase-3 antineutrophil cytoplasmic antibodies (ANCA), involving the lungs, left orbit, and nasopharynx. He had received 3 courses of monthly intravenous cyclophosphamide (cumulative dose: $2.25 \mathrm{~g}$ ). Prednisolone 25 mg per day (cumulative dose: $680 \mathrm{mg}$ ) and trimethoprim/sulfamethoxazole at a prophylactic dose had been prescribed. Plain chest and abdomen radiographs and a chest computed tomography (CT) scan revealed increased size of the cavitary lung mass, nodular consolidation, and new onset of pneumomediastinum and pneumoperitoneum (Figure $1 \mathrm{~A} \sim \mathrm{C}$ ). Subsequent abdominal CT images with the lung window setting showed a large amount of intramural air on the wall of the ascending colon, and pneumatosis intestinalis (PI) was diagnosed (Figure 1D). The patient did not complain of abdominal pain. Intravenous antibiotics along with fasting, fluid supplementation, and oxygen therapy was maintained for 3 days. However, the amount of intramural air on the ascending colon and pneumomediastinum did not change in follow-up plain radiographs. Meanwhile, the presence of blood tinged sputum continued, and the proteinase-3 ANCA titer (cut-off $5 \mathrm{U} / \mathrm{ml}$; Orgentec, Mainz, Germany) was elevated compared to a previous study (from $19.0 \mathrm{U} / \mathrm{ml}$ to $61.3 \mathrm{U} / \mathrm{ml}$ ). All blood and sputum culture sets were reported as "no growth." The prednisolone dose was increased to $1 \mathrm{mg} / \mathrm{kg}$, leading to gradual improvement of intramural air on the ascending colon and blood tinged sputum. The patient was able to tolerate a regular diet and was discharged in stable condition. An additional set of plain radiographs of the chest and abdomen and CT scan obtained 4 weeks later revealed that pneumomediastinum and intestinal gas accumulation had completely resolved (Figure $1 \mathrm{E} \sim \mathrm{H}$ ).

$\mathrm{PI}$ is defined as the presence of gas within the wall of the gastrointestinal tract [1]. It has been reported in various pulmonary, intestinal bowel, infectious, and autoimmune diseases [2]. In addition, medication-associated or postoperative etiologies have been described [2]. Treatment with glucocorticoid could be associated with development of PI in patients with ANCA-associated vasculitis [3]. In this case, PI appeared simultaneously with hemoptysis, which is considered an aggravation of granulomatosis with polyangiitis, over the course of prednisolone tapering. The rapid radiographic improvement after increasing the dose of prednisolone, without evidence of infection in this patient, supports the hypothesis that PI was associated with exacerbation of vasculitis. A vasculitis-based pathogenesis within the intestinal territory has been proposed as a cause for development of PI among patients with systemic lupus erythematosus and systemic necrotizing vasculitis $[4,5]$. While many patients with autoimmune disease who develop PI respond to conservative therapy and have a benign course, it is prudent for rheumatologists caring for such patients to consider PI in the differential diagnosis. The treatment decision of PI should be based on the patient's clinical features and underlying etiologies since treatment of the underlying condition often improves PI, while glucocorticoids can often exacerbate PI.

Received : April 27, 2020, Revised : May 22, 2020, Accepted : June 4, 2020

Corresponding to : Jung Hee Koh (D)http://orcid.org/0000-0002-6617-1449

Division of Rheumatology, Department of Internal Medicine, Bucheon St. Mary's Hospital, College of Medicine, The Catholic University of Korea, 327 Sosa-ro, Wonmi-gu, Bucheon 14647, Korea. E-mail : jungheekoh@gmail.com 

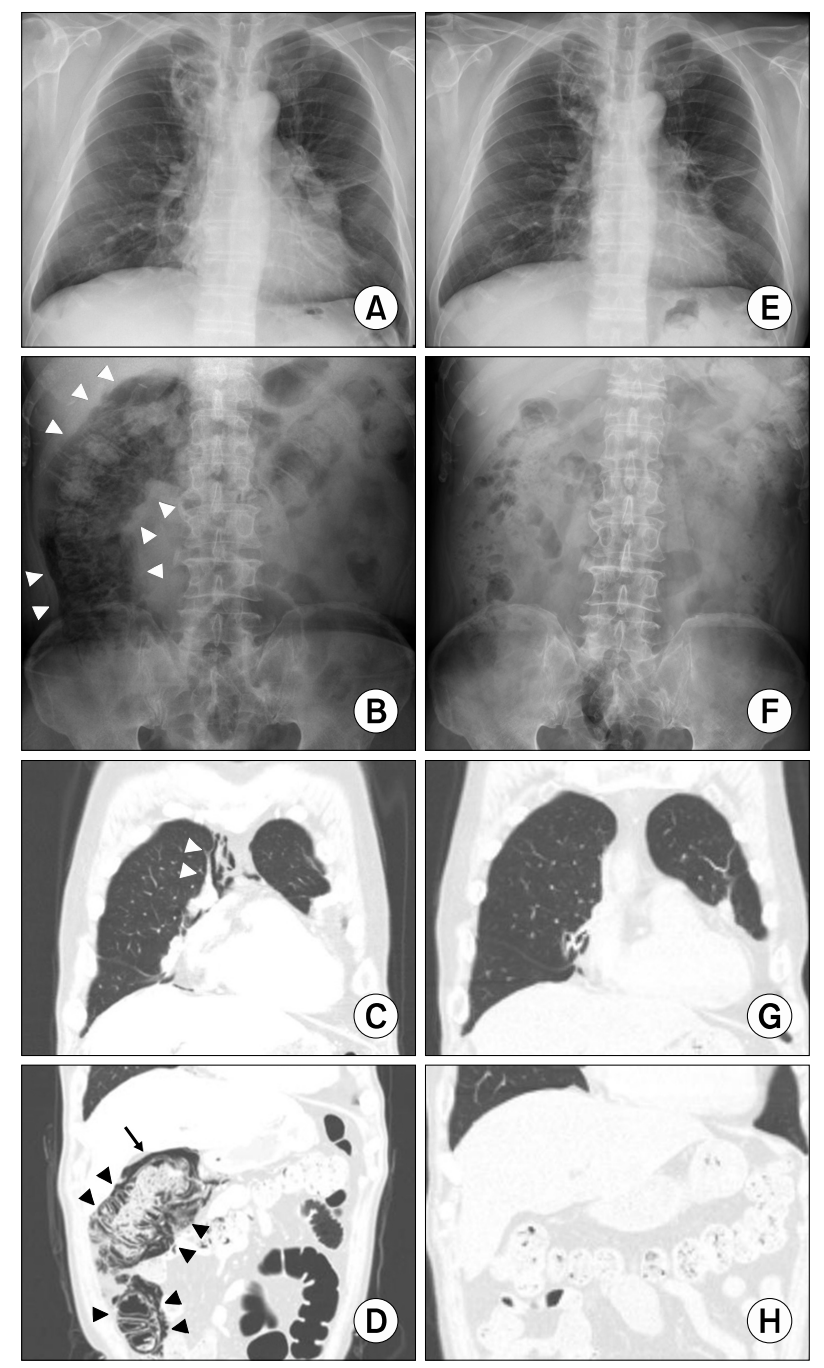

Figure 1. Plain radiographs and computed tomography (CT) scans at the time of pneumatosis intestinalis and follow-up after 4 weeks from the onset. Chest radiograph on admission (A). Supine abdominal radiograph showing linear pneumatosis intestinalis of the colon (arrowheads) (B). The coronal CT scan (C) of the chest shows pneumomediastinum (arrowheads). The coronal abdominal CT images using the lung window setting (D) show free air (long arrows) and colon pneumatosis intestinalis (arrowheads). Follow-up plain radiographs (E and F) and $C T$ scan using the lung window setting $(G$ and $H)$ show the complete resolution of pneumomediastinum and pneumatosis intestinalis.

\section{CONFLICT OF INTEREST}

No potential conflict of interest relevant to this article was reported.

\section{AUTHOR CONTRIBUTIONS}

J.H.K. had full access to all data in the study and takes responsibility for the integrity of the data and accuracy of the data analysis. J.H.K. and A.K. contributed to the study concept, design, data acquisition, analysis and interpretation. J.H.K. and A.K. draft the manuscript and contributed to the critical revision. All authors read and approved the final manuscript.

\section{REFERENCES}

1. Ho LM, Paulson EK, Thompson WM. Pneumatosis intestinalis in the adult: benign to life-threatening causes. AJR Am J Roentgenol 2007;188:1604-13.

2. Khalil PN, Huber-Wagner S, Ladurner R, Kleespies A, Siebeck M, Mutschler W, et al. Natural history, clinical pattern, and surgical considerations of pneumatosis intestinalis. Eur J Med Res 2009;14:231-9.

3. Nakagawa S, Akimoto T, Takeda S, Okada M, Miki A, Yamamoto H, et al. Antineutrophil cytoplasmic antibodyassociated glomerulonephritis complicated by pneumatosis intestinalis. Clin Med Insights Case Rep 2015;8:65-70.

4. Mizoguchi F, Nanki T, Miyasaka N. Pneumatosis cystoides intestinalis following lupus enteritis and peritonitis. Intern Med 2008;47:1267-71.

5. Pagnoux C, Mahr A, Cohen P, Guillevin L. Presentation and outcome of gastrointestinal involvement in systemic necrotizing vasculitides: analysis of 62 patients with polyarteritis nodosa, microscopic polyangiitis, Wegener granulomatosis, Churg-Strauss syndrome, or rheumatoid arthritis-associated vasculitis. Medicine (Baltimore) 2005;84: 115-28. 